\title{
Additive fault tolerant control of nonlinear singularly perturbed systems against actuator fault
}

\author{
Adel Tellili, ${ }^{*}{ }^{* *}$ Aymen Elghoul, \\ Mohamed Naceur Abdelkrim*,***
}

\begin{abstract}
This paper presents the design of an additive fault tolerant control for nonlinear time-invariant singularly perturbed systems against actuator faults based on Lyapunov redesign principle. The overall system is reduced into subsystems with fast and slow dynamic behavior using singular perturbation method. The time scale reduction is carried out when the singular perturbation parameter is set to zero, thus avoiding the numerical stiffness due to the interaction of two different dynamics. The fault tolerant controller is computed in two steps. First, a nominal composite controller is designed using the reduced subsystems. Secondly, an additive part is combined with the basic controller to overcome the fault effect. The derived $\varepsilon$ independent controller guarantees asymptotic stability despite the presence of actuator faults. The Lyapunov stability theory is used to prove the stability provided the singular perturbation parameter is sufficiently small. The theoretical results are simulated using a numerical application.
\end{abstract}

K e y w ords: nonlinear time-invariant singularly perturbed systems, singular perturbation method, additive fault tolerant control, actuator defect, Lyapunov theory

\section{Introduction}

Singularly perturbed systems belong to a class of systems having a mathematical model which contains a set of differential equations depending on a small positive parameter, called parameter of singular perturbation, multiplying the derivative terms of a part of the equations. They are often occur in the physical systems in engineering field, like power systems, dynamic networks, robots, energy and particles transfer mechanism and so on. Such processes involve interconnected slow and fast dynamics which lead commonly to numerical stiffness in the control design methods. To overcome such problems, the methodology of singular perturbation, based on reduction techniques, is frequently used in the literature. The principle is to decompose the overall system into two subsystems with slow and fast dynamics, so that the actual controller, which depends on the parameter of the singular perturbation, is approached by a composite form of the controllers stabilizing the decomposed subsystems [1-4].

Like other systems, the multi time scale systems can be affected by faults that may prevail the controllers, the actuators, or other system components. In this case, an appropriate control strategy, called fault tolerant or reconfigurable control, is required to guarantee nominal performances despite the faults occurrence. Such control schemes are able to recover system and component performances in case of fault happening and to surmount the constraints caused by the typical control scheme.
The aim of this control scheme is to preserve security of machines and system operators [5-9]. For nonlinear systems, meaningful results are presented in the literature. Two different methods are presented. In passive fault tolerant controller design methods, the controller parameters remain unchangeable throughout the faulty and the fault free case. In this situation, techniques like HamiltonJacobi inequality approach and robust pole region assignment method are used $[10,11]$. The active methods like sliding mode-based control methods and adaptive backstepping compensation control, by contrast, need an online fault detection scheme to achieve fault diagnosis step, and then a procedure to ensure the compensation of detected faults $[12,13]$. Diverse techniques are proposed in the literature to design fault tolerant controllers for nonlinear systems. Liang and $\mathrm{Xu}$ in [14] proposed a variable structure stabilizing control law to tolerate the presence of actuator fault in a nonlinear system. The derived control scheme doesn't require the solution of HamiltonJacobi inequality. Benosman and Lum in [15] developed a Lyapunov-based passive feedback controller to guarantee stability of nonlinear affine system with actuator faults. Ma and Yang in [16] designed a fault diagnosis procedure and an active reconfigurable control strategy for nonlinear uncertain dynamic system against time-varying actuator fault. An improved high gain observer is realized to supply more on-line information to generate the reconfigurable controller.

\footnotetext{
* Research Unit MACS, Higher National Engineering School at Gabes, Tunisia; ${ }^{* *}$ Higher Institute of Technological Studies at Djerba (ISETJB), Tunisia; ${ }^{* * *}$ National Engineering School at Gabes, University of Gabes, Tunisia; naceur.abdelkrim@enig.rnu.tn, aymengoul@hotmail.fr, Adel.Tellili@isetjb.rnu.tn
} 
On the other hand, several approaches to control multi time scale systems have been proposed. Especially, in linear case, Li et al in [17] derived a linear quadratic control scheme for singularly perturbed systems. The designed controller is reliable to actuator failures; it is based on fast and slow sub-controllers so that it becomes independent of the singular perturbation parameter. In [18], Tellili et al proposed a reconfigurable adaptive method to compensate for any defects that may affect the sensors and the actuators in presence of external perturbation. In both cases, sensor and actuator fault, a controller for the original system was designed and then simplified using singular perturbation method. In nonlinear case, reconfigurable control was investigated by some authors in case of systems undergoing actuator saturation [19-21]. In particular, the methods used to control nonlinear timeinvariant singularly perturbed systems did not consider actuator additive faults.

The principal contribution of this work is to develop a control strategy for nonlinear time-invariant singularly perturbed systems affected by actuator additive faults, without going through the linearization of the nonlinear system. The control scheme is a combination of a composite control and an additive part used to accommodate the presence of a fault. The singular perturbation method will be exploited to avoid the numerical stiffness induced by the interconnection of the fast and slow dynamics. Thereby, the reliable controller will be independent of the singular perturbation parameter.

The succeeding paper sections are arranged as follows. In Section 2, the system structure and the problem formulation will be depicted. The main results are formulated in Section 3. An application in form of a simulation is illustrated in Section 4. Finally, a conclusion ends the paper.

\section{System characterization and problem formulation}

The following nonlinear two-time scales time-invariant singularly perturbed system will be considered $[22,23]$

$$
\begin{aligned}
\dot{x}(t) & =f_{1}(x, y)+g_{1}(x, y) u(t) \\
\varepsilon \dot{y}(t) & =f_{2}(x, y)+g_{2}(x, y) u(t)
\end{aligned}
$$

where $x \in B_{x} \subset \Re^{n 1}$ and $y \in B_{y} \subset \Re^{n 2}$ represent the state vectors, $u \in \Re^{m}$ corresponds to the control vector. For $i=1,2, f_{i}$ and $g_{i}$ are locally Lipschitz in a field enclosing the origin and $f_{i}$ satisfies $f_{i}(0,0)=0$. The scalar $\varepsilon$ is a singular perturbation parameter taking values between 0 and 1 ; it characterizes the time scale separation between the slow and the fast dynamics. $(x, y)=(0,0)$ is assumed to be an isolated equilibrium state.

The singular perturbation theory will be used to approximate the slow and fast dynamics by setting $\varepsilon=0$ in the $\dot{y}$-equation and solving for $y$ in terms of $x[1,24,25]$. It follows for the second equation in system (1)

$$
0=f_{2}(x, y)+g_{2}(x, y) u(t) \text {. }
$$

The singularly perturbed system is supposed to be standard, which means, the equation (2) has only a solution $y=h\left(x, u_{s}\right)$, where $u_{s}$ is the slow part of the control $u$. Substituting this solution into the first equation of system (1), the following reduced slow subsystem is obtained

$$
\dot{x}(t)=f_{1}\left(x, h\left(x, u_{s}\right)\right)+g_{1}\left(x, h\left(x, u_{s}\right)\right) u_{s}(t) .
$$

It is assumed that only the origin of the closed-loop slow subsystem (3) is an asymptotically stable equilibrium, so there exists a feedback slow control law $u_{s}(t)=p_{s}(x)$, where $p_{s}$ is locally Lipschitz vector function, that renders the slow dynamics asymptotically stable; we consider also a positive definite Lyapunov function $V_{s}(x)$ guaranteeing for all $x \in B_{x}$,

$$
\begin{aligned}
& \frac{\partial V_{s}(x)}{\partial x}\left[f_{1}\left(x, h\left(x, u_{s}\right)\right)+\right. \\
& \left.\qquad g_{1}\left(x, h\left(x, u_{s}\right)\right) u_{s}\right] \leq-a L_{s}^{2}(x)
\end{aligned}
$$

Where $a \succ 0$ and $L_{s}(x)$ is a positive definite function. The reduced fast subsystem is generated by

$$
\frac{\mathrm{d} y}{\mathrm{~d} \tau}=f_{2}(x, y)+g_{2}(x, y)\left(u_{s}+u_{f}\right)
$$

in which the fast time scale is defined as $\tau=t / \varepsilon$ and $x$ is assumed to be a constant parameter equal to its initial value. There exists a feedback fast control law $u_{f}(t)=p_{f}(x, y)$ which asymptotically stabilizes the fast dynamics, such that the equilibrium $y=h\left(x, u_{s}\right)$ of the closed loop fast subsystem is supposed asymptotically stable uniformly in $x \in \Re^{n 1}$. The fast controller satisfies $p_{f}\left(x, h\left(x, p_{s}(x)\right)=0\right.$, where $p_{f}$ is locally Lipschitz vector function. The composite control of the global system is expressed as a sum of the fast and slow subcontrollers $[1,25,26]$

$$
u=u_{s}(x)+u_{f}(x, y) .
$$

The composite feed-back control is designed so that the origin is an asymptotically stable equilibrium of the singularly perturbed closed-loop system (1). For the fast subsystem $(5), V_{f}(x, y)$ is defined as a positive definite Lyapunov function such that for all $(x, y) \in B_{x} \times B_{y}$

$$
\begin{aligned}
\frac{\partial V_{f}}{\partial y}\left[f_{2}(x, y)+g_{2}(x, y)\left(p_{s}+p_{f}\right)\right] & \leq \\
& -b L_{f}^{2}\left(y-h\left(x, p_{s}\right)\right)
\end{aligned}
$$

where $b \succ 0$ and $L_{f}$ is a positive definite function.

A composite Lyapunov function candidate for the singularly perturbed system (1) is defined by a weighted sum 
of the Lyapunov functions for the reduced fast and slow subsystems, so that

$$
W(x, y)=(1-d) V_{s}+d V_{f}
$$

where $0 \prec d \prec 1$ is a free parameter to be chosen.

The composite Lyapunov function $w(x, y)$ will be derived along the trajectories of (1):

$$
\begin{aligned}
\frac{\partial W(x, y)}{\partial t}=(1 & -d) \frac{\partial V_{s}(x)}{\partial x} \frac{\partial x}{\partial t} \\
& +d\left(\frac{\partial V_{f}(x, y)}{\partial x} \frac{\partial x}{\partial t}+\frac{\partial V_{f}(x, y)}{\partial y} \frac{\partial y}{\partial t}\right)
\end{aligned}
$$

Using equation (1), we get

$$
\begin{aligned}
\frac{\partial W(x, y)}{\partial t}= & (1-d) \frac{\partial V_{s}(x)}{\partial x}\left[f_{1}(x, y)+g_{1}(x, y) u\right] \\
+ & \frac{d}{\varepsilon} \frac{\partial V_{f}(x, y)}{\partial y}\left[f_{2}(x, y)+g_{2}(x, y) u\right] \\
& +d \frac{\partial V_{f}(x, y)}{\partial x}\left[f_{1}(x, y)+g_{1}(x, y) u\right] .
\end{aligned}
$$

After some algebraic manipulations, (10) can be expressed as

$$
\begin{aligned}
\frac{\partial W(x, y)}{\partial t}= & \frac{d}{\varepsilon} \\
& \frac{\partial V_{f}(x, y)}{\partial y}\left[f_{2}(x, y)+g_{2}(x, y) u\right] \\
& +(1-d) \frac{\partial V_{s}(x)}{\partial x}\left[f_{1}\left(x, h\left(x, u_{s}\right)\right)\right. \\
& \left.+g_{1}\left(x, h\left(x, u_{s}\right)\right) u_{s}\right]+T\left(x, y, u, u_{s}\right)
\end{aligned}
$$

where

$$
\begin{aligned}
& T\left(x, y, u, u_{s}\right)=(1-d) \frac{\partial V_{s}(x)}{\partial x}\left[f_{1}(x, y)+g_{1}(x, y) u\right. \\
& \left.-f_{1}\left(x, h\left(x, u_{s}\right)\right)-g_{1}\left(x, h\left(x, u_{s}\right)\right) u_{s}\right] \\
& +d \frac{\partial V_{f}(x, y)}{\partial x}\left[f_{1}(x, y)+g_{1}(x, y) u\right] .
\end{aligned}
$$

The first and the second term of the equation (11) represent the derivatives of $V_{f}$ and $V_{s}$ along the trajectories of the fast and slow subsystems respectively. From equations (4) and (7), we can see that these two terms are negative definite in $x$ and $y$. The last term $T\left(x, y, u, u_{s}\right)$ represents the impact of the interconnection among the fast and slow dynamics. This term can be neglected if singular perturbation parameter $\varepsilon$ remains small [27].

Using inequalities (4) and (7), equation (11) can be rearranged further to get

$$
\frac{\partial W(x, y)}{\partial t} \leq-N(x, y)
$$

where $N(x, y)=a L_{s}^{2}(x)+\frac{d}{\varepsilon} b L_{f}^{2}\left(y-h\left(x, p_{s}\right)\right)$ is positive definite. Consequently, the composite control (6) ensures that the closed-loop system (1) admits an asymptotically stable equilibrium at the origin for a given interval of the parameter $\varepsilon$ and $w(x, y)$ is a Lyapunov function of this system $[1,24,28]$.

\section{Principal results}

In the following, the essential results will be presented. Suppose that the reduced fast and slow subsystems are stabilizable in the domain $B_{x} \times B_{y}$ and the state $(x, y)$ is accessible for feedback, we seek for a control scheme in order to stabilize asymptotically the point $(x=0$, $y=0$ ) of the closed-loop overall two time scales singularly perturbed system despite actuator fault occurrence.

The system (1), where the actuator is affected with additive fault, can be expressed as

$$
\begin{aligned}
\dot{x}(t) & =f_{1}(x, y)+g_{1}(x, y)(u+D(t, x, y)), \\
\varepsilon \dot{y}(t) & =f_{2}(x, y)+g_{2}(x, y)(u+D(t, x, y)
\end{aligned}
$$

where $D(t, x, y)$ represents an actuator fault which verifies $\|D(t, x, y)\| \leq B(t, x, y)$ and $B(t, x, y)$ is a nonnegative continuous function. The controller takes the form

$$
u=u_{\text {nom }}+u_{\text {add }}
$$

where $u=u_{\text {nom }}$ represents the nominal controller that stabilizes the overall system in case of a faultless actuator, it corresponds to the composite controller (6). $u_{\text {add }}$ denotes the additive part to be designed in order to remove the fault effect in the actuator. The proposed fault tolerant controller to stabilize the faulty system (13) is

$$
u=u_{\text {nom }}+u_{\text {add }}
$$

where

$$
u_{\mathrm{add}}=-B \frac{(1-d) \frac{\partial V_{s}(x)}{\partial x} g_{1}+d \frac{\partial V_{f}(x, y)}{\partial y} g_{2}}{\left\|(1-d) \frac{\partial V_{s}(x)}{\partial x} g_{1}+d \frac{\partial V_{f}(x, y)}{\partial y} g_{2}\right\|},
$$

$(\cdot)^{\top}$ denotes the transpose of $(\cdot)$ and $\|(\cdot)\|$ the Euclidian norm of $(\cdot)$.

The next theorem is presented to accomplish the control of the nonlinear singularly perturbed system (13) even when some actuators operate abnormally.

THEOREM 1. The faulty nonlinear singularly perturbed system given by (13) is considered. Suppose that the reduced fast and slow subsystems are stabilizable and the actuator fault is bounded. Then there exists a singular perturbation parameter $\varepsilon^{*}>0$ such that for all $\varepsilon \in\left(0, \varepsilon^{*}\right]$, the origin $(x, y)=(0,0)$ of the faulty nonlinear singularly perturbed system is locally asymptotically stable under the fault tolerant control law (15) even when the actuators undergo abnormal operation.

P r o o f . According to equations (13) and (14), it follows for the closed-loop faulty nonlinear singularly perturbed system

$$
\begin{aligned}
\dot{x}(t) & =f_{1}(x, y)+g_{1}(x, y)\left(u_{\mathrm{nom}}+u_{\text {add }}+D(t, x, y)\right), \\
\varepsilon \dot{y}(t) & =f_{2}(x, y)+g_{2}(x, y)\left(u_{\mathrm{nom}}+u_{\text {add }}+D(t, x, y)\right) .
\end{aligned}
$$


An $\varepsilon$-dependent Lyapunov function candidate will be designed,

$$
W(\varepsilon, x, y)=(1-d) V_{s}+\varepsilon d V_{f}
$$

The derivative of $W(\varepsilon, x, y)$ will be established along the trajectories of system (16), this leads to

$$
\begin{gathered}
\frac{\partial W(\varepsilon, x, y)}{\partial t}=d \frac{\partial V_{f}(x, y)}{\partial y}\left[f_{2}+g_{2}\left(u_{\mathrm{nom}}+u_{\mathrm{add}}+D\right)\right] \\
+(1-d) \frac{\partial V_{s}(x)}{\partial x}\left[f_{1}+g_{1}\left(u_{\mathrm{nom}}+u_{\mathrm{add}}+D\right)\right] \\
+d \varepsilon \frac{\partial V_{f}(x, y)}{\partial x}\left[f_{1}+g_{1}\left(u_{\mathrm{nom}}+u_{\mathrm{add}}+D\right)\right] .
\end{gathered}
$$

The isolation of the terms depending on the nominal control yields the following system

$$
\begin{gathered}
\frac{\partial W(\varepsilon, x, y)}{\partial t}=d \frac{\partial V_{f}(x, y)}{\partial y}\left(f_{2}+g_{2} u_{\mathrm{nom}}\right) \\
+(1-d) \frac{\partial V_{s}(x)}{\partial x}\left(f_{1}+g_{1} u_{\mathrm{nom}}\right)+d \varepsilon \frac{\partial V_{f}(x, y)}{\partial x}\left(f_{1}+g_{1} u_{\mathrm{nom}}\right) \\
+(1-d) \frac{\partial V_{s}(x)}{\partial x} g_{1}\left(u_{\mathrm{add}}+D\right)+d \frac{\partial V_{f}(x, y)}{\partial y} g_{2}\left(u_{\mathrm{add}}+D\right) \\
+d \varepsilon \frac{\partial V_{f}(x, y)}{\partial x} g_{1}\left(u_{\mathrm{add}}+D\right)
\end{gathered}
$$

Set $R_{1}^{\top}=\frac{\partial V_{s}(x)}{\partial x} g_{1}, R_{2}^{\top}=\frac{\partial V_{f}(x, y)}{\partial y} g_{2}, R_{3}^{\top}=\frac{\partial V_{f}(x, y)}{\partial x} g_{1}$ and taking into account inequality (12), the equation takes the form

$$
\begin{aligned}
\frac{\partial W(\varepsilon, x, y)}{\partial t} \leq & -N(x, y)+(1-d) R_{1}^{\top}\left(u_{\mathrm{add}}+D\right) \\
& +d R_{2}^{\top}\left(u_{\mathrm{add}}+D\right)+d \varepsilon R_{3}^{\top}\left(u_{\mathrm{add}}+D\right)
\end{aligned}
$$

which can be rearranged in the following inequality

$$
\begin{aligned}
& \frac{\partial W(\varepsilon, x, y)}{\partial t} \leq-N(x, y)+\left((1-d) R_{1}^{\top}+d R_{2}^{\top}\right. \\
& \left.\quad+d \varepsilon R_{3}^{\top}\right) u_{\mathrm{add}}+\left((1-d) R_{1}^{\top}+d R_{2}^{\top}+d \varepsilon R_{3}^{\top}\right) D .
\end{aligned}
$$

The term $N(x, y)$ is defined as in (12). Using the singular perturbation procedure by setting the singular perturbation parameter $\varepsilon$ to zero and considering the upper bound of the fault leads to the following expression

$$
\begin{aligned}
\frac{\partial W(0, x, y)}{\partial t} \leq-N(x, y) & +\left((1-d) R_{1}^{\top}+d R_{2}^{\top}\right) u_{\text {add }} \\
& +\left\|\left((1-d) R_{1}^{\top}+d R_{2}^{\top}\right)\right\| B
\end{aligned}
$$

The term $N(x, y)$ is due to the nominal composite control of the fault-free overall system, this term is positive definite in $x$ and $y$ by (12). Whereas the second and third terms designate, respectively, the effect of the additive control $u_{\text {add }}$ and the fault $D(t, x, y)$ on $\dot{W}(x, y)$. Consequently, $u_{\text {add }}$ will be chosen to remove the influence of the actuator fault $D(t, x, y)$ on $\dot{W}(x, y)$ so that the right term of inequality (22) remains negative definite.
In view of the control law (15) and taking into account the assumptions about the fault, it is obvious that $\dot{W}(x, y) \leq 0$. Thus, it can be concluded that the origin $(x, y)=(0,0)$ of the faulty overall system is a locally asymptotically stable equilibrium point for the system (16) under the fault tolerant control law (15) for any singular perturbation parameter $\varepsilon \in\left(0, \varepsilon^{*}\right]$.

R e m a r k . The discontinuity of the control law (15) may engender chattering effect. This problem is commonly overcome by approximating the discontinuous function by a saturation function.

\section{Example of application}

To depict the efficiency of the studied reconfigurable control scheme, the following nonlinear singularly perturbed system is considered

$$
\begin{aligned}
\dot{x} & =y-x, \\
\varepsilon \dot{y} & =-x-e^{y}+1+u .
\end{aligned}
$$

The reduced slow subsystem is established by letting $\varepsilon=$ 0 in (23). It takes the form

$$
\dot{x}=\log _{e}\left(-x+1+u_{s}\right)-x
$$

and the slow part of the state $y$ is expressed as

$$
y_{s}=\log _{e}\left(-x+1+u_{s}\right) \text {. }
$$

It is easy to deduce that the control of the slow subsystem $u_{s}=x-1+e^{-x}$ ensures that the closed-loop reduced slow subsystem (24) becomes asymptotically stabilizing about the origin. A corresponding slow Lyapunov function candidate is $V_{s}=0.5 x^{2}$. The reduced fast subsystem is given by

$$
\frac{\mathrm{d} y}{\mathrm{~d} \tau}=-x-e^{y}+1+u_{s}+u_{f}
$$

Taking into account the above mentioned slow control, the fast control $u_{f}=e^{y}-e^{-x}-y_{f}$ stabilizes the state $y$ about the origin. $y_{f}$ is the fast part of the state $y$, such that $y_{f}=y-y_{s}$. A corresponding fast Lyapunov function candidate is $V_{f}=0.5 y_{f}^{2}=0.5(x+y)^{2}$. The composite control, considered as the nominal control of the fault free overall system, will be designed as the sum of the slow and fast sub-controllers, taking the following form

$$
u_{\mathrm{comp}}=u_{s}+u_{f}=e^{y}-y-1 .
$$

The resulting closed-loop overall system, after substituting of (27) in (23) is

$$
\begin{aligned}
\dot{x} & =-x+y, \\
\varepsilon \dot{y} & =-x-y
\end{aligned}
$$



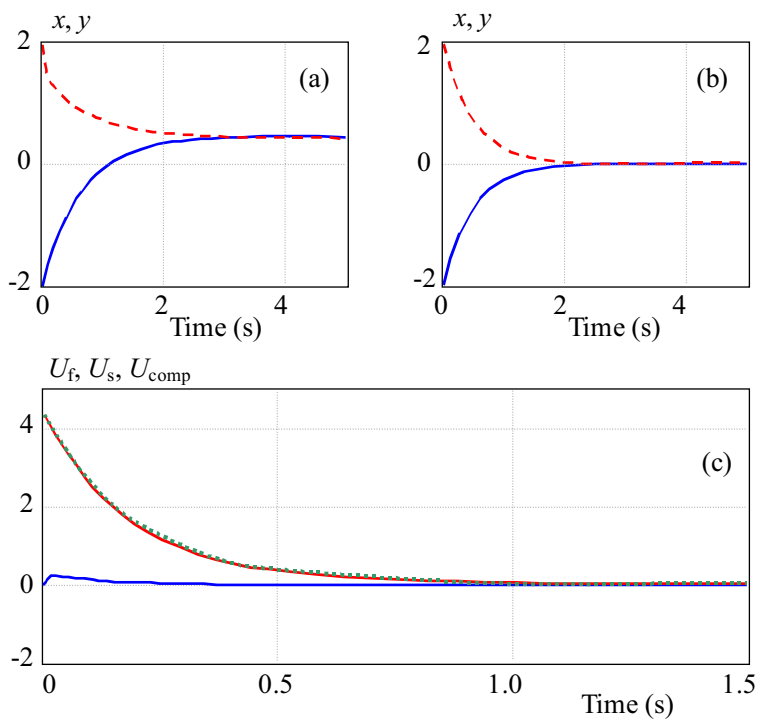

Fig. 1. States trajectories in fault-free case. (a) $-x$ (dashed line) and $y$ (solid line) in open-loop, (b) - States in closed-loop with composite control, (c) - fast (-.), slow (--) and composite (-) controllers by $\varepsilon=0.01$
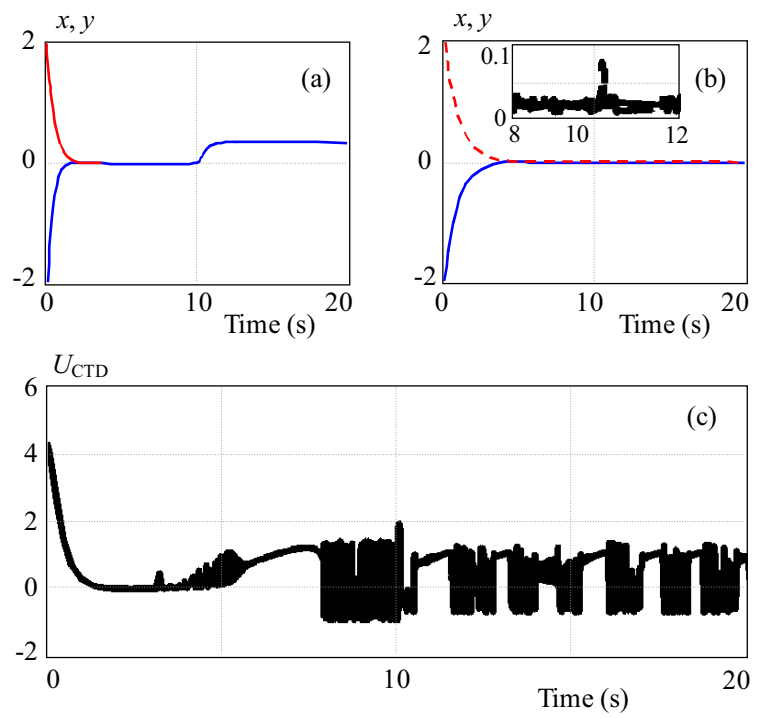

Fig. 2. States trajectories after the appearance of the actuator fault. (a) - $x$ (dashed line) and $y$ (solid line) in case of nominal control, (b) - states in case of fault tolerant control, (c) — fault tolerant control controller by $\varepsilon=0.01$

where the real parts of the eigenvalues remain negative. This concludes that the origin of system (28) gets an asymptotically stable equilibrium of the closed-loop system. The simulation results in Fig. 1 show the composite control and the states trajectories in the fault-free case, starting from $\left(x_{0}, y_{0}\right)=(-2,2)$. It is clear that the composite control (27) ensures the asymptotical stability of the origin.

However, the occurrence of constant additive actuator fault of amplitude 0.7 , at time instance $10 \mathrm{sec}$, yields a loss of the actuator performance. Consequently, the states drive to another stationary point (see Fig. 2(a)). This means that the composite controller is not able to stabilize the origin equilibrium point in the faulty case.
Next, a fault tolerant control scheme will be proposed, according to the equation (15), to compensate for the actuator fault, it takes the form

$$
u=u_{\mathrm{comp}}+u_{\mathrm{add}}=e^{y}-y-1-0.7 \frac{x+y}{\|x+y\|} .
$$

Figure 2(b) depicts that the states deviation is corrected using the fault tolerant control (28) and the singularly perturbed system stabilizes at the origin equilibrium point, despite the presence of actuator faults. The zoom in Fig. 2(b) illustrates the effect of fault on the states when the fault tolerant control is used. Figure 2(c) shows the corresponding fault tolerant control which presents high chattering effect.

To solve this problem, the discontinuous function will be changed with a saturation function. The simulation results in Fig. 3(a) and (b) represent, respectively, the states and the controller after the substitution of the discontinuity in the control law. It is clear that the chattering effect is reduced and the states remain at the same equilibrium point.

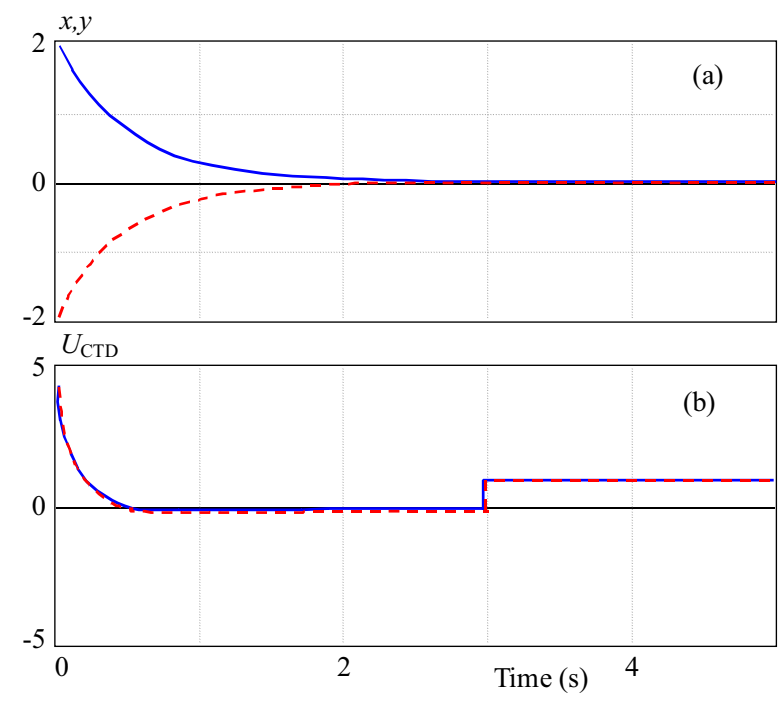

Fig. 3. States trajectories (a) - and fault tolerant control (b) after the attenuation of chattering effect

\section{Conclusion}

The control for nonlinear singularly perturbed systems subject to actuator fault is considered. The control scheme involves two parts. First, a composite controller, depending on fast and slow sub-controllers, is designed to treat the nominal case. The second part is generated to deal with actuator faults in additive form. The Lyapunov function for the overall system is designed in composite weighted form using the slow and fast local Lyapunov functions. To ensure the stability in presence of additive 
fault, which means that the time derivative of the Lyapunov functions is negative definite, an additive controller will be designed using slow and fast subsystems. A further extension to this work can be the examination of other forms of faults like loss of effectiveness in presence of external perturbation and the consideration of non standard singularly perturbed systems.

In the illustrative example, it is shown that the composite control was unable to hold the origin as an asymptotically stable equilibrium of the overall system, whereas, the use of the reconfigurable control eliminates the effect of actuator failure from the states trajectories.

\section{REFERENCES}

[1] P. Y. Kokotovic, H. K. Khalil, J. O'reilly, Singular Perturbation Methods in Control Analysis and Design, Harcourt Brace Jovanovich publishers, Academic press, United States of America, 1999.

[2] P. Y. Kokotovic, R. E. O'malley Jr, P. Sannuti, "Singular Perturbations and Order Reduction in Control Theory - an Overview", Automatica, vol. 12, no. 2, Mar 1976, 123-132.

[3] A. Massoum, A. Meroufel, P. Wira, M. K. Fellah, "Fuzzy Control of the Permanent Magnet Synchronous Generator Machine Singularly Perturbed Fed by a Three Level Inverter", Journal of Electrical Engineering, vol. 63, no. 3, Mar 2012, 186-190.

[4] W. H. Chen, G. Yuan, W. X. Zheng, "Robust Stability of Singularly Perturbed Impulsive Systems Under Nonlinear Perturbation", IEEE Transactions on Automatic Control, vol. 58, no. 1, Jan 2013, 168-174.

[5] D. Bustan, S. K. H. Sani, N. Pariz, "Adaptive Fault-tolerant Spacecraft Attitude Control Design with Transient Response Control", IEEE/ASME Transactions on Mechatronics, vol. 19, no. 4, Aug 2014, 1404-1411.

[6] Y. Wang, P. Zhou, Q. Wang, D. Duan, "Reliable Robust Sampled-Data H-infinity Output Tracking Control with Application to Flight Control", Information Technology and Control, vol. 43, no. 2, 2014, 175-182.

[7] R. J. Patton, "Fault-Tolerant Control Systems: the 1997 Situation", Proceedings of the Safe'Process, United Kingdom, 1997, $1033-1055$.

[8] M. Boukhnifer, "Active Fault Tolerant Control for Ultrasonic Piezoelectric Motor", Journal of Electrical Engineering, vol. 63, no. 4, July 2012, 224-232.

[9] M. S. Khireddine, A. Boutarfa, "Reconfigurable Control for a Scara Robot Using RBF Networks", Journal of Electrical Engineering, vol. 61, no. 2, Mar 2010, 100-106.

[10] G. H. Yang, S. Y. Zhang, J. Lam, J. L. Wang, "Reliable Control Using Redundant Controllers", IEEE Transactions on Automatic Control, vol. 43, no. 11, Nov 1998, 1588-1593.

[11] Q. Zhao, J. Jiang, "Reliable State Feedback Control System Design Against Actuator Failures", Automatica, vol. 34, no. 10, Oct 1998 1267-1272.

[12] M. L. Corradini, G. Orlando, "Actuator Failure Identification and Compensation Through Sliding Modes", IEEE Transactions on Control Systems Technology, vol. 15, no. 1, Jan 2007, 184-190.
[13] W. Wang, C. Y. Wen, "Adaptive Compensation for Infinite Number of Actuator Failures or Faults", Automatica, vol. 47, no. 10, Aug 2011, 2197-2210.

14] Y.-W. Liang, S.-D. Xu, "Reliable Control of Nonlinear Systems via Variable Structure Scheme", IEEE Transactions on Automatic Control, vol. 51, no. 10, Oct 2006, 1721-1726.

[15] M. Benosman, K.-Y. Lum, "Passive Actuators' Fault-Tolerant Control for Affine Nonlinear Systems", IEEE Transactions on Automatic Control, vol. 18, no. 1, Jan 2010, 152-163.

[16] H. J. Ma, G. H. Yang, "Detection and Adaptive Accommodation for Actuator Faults of a Class of Nonlinear Systems", IET Control Theory and Applications, vol. 6, no. 14, Sep 2012, 2292-2307.

[17] Y. Li, J. L. Wang, G. H. Yang, "Reliable Linear-Quadratic Control for Singularly Perturbed Systems", International Journal of Systems Science, vol. 33, no. 12, 2002, 949-958.

[18] A. Tellili, N. Abdelkrim, A. Challouf, M. N. Abdelkrim, "Reconfigurable Control of Two-Time Scale Systems in Presence of Additive Faults", Information Technology and Control, vol. 44, no. 4, 2015, 357-366.

Received 23 November 2016

Adel Tellili was born in Tunisia in 1967, he graduated from the Technical University of Karlsruhe, Germany, in 1994. He received the Master degree in Automation in 2003 from the National School of Engineers of Sfax (ENIS), Tunisia and the PhD degree from the same school in January 2007. He is currently an Assistant Professor at the Higher Institute of Technological Studies at Djerba. He is member at research Laboratory on Modeling, Analysis and Control of Systems (MACS). His research interests include singularly perturbed systems, fault diagnosis and fault tolerant control.

Aymen Elghoul was born in Tunisia in 1987, he graduated from the National School of Engineers in Carthage, Tunisia, in 2013. He received the Master degree from the Higher Institute of Industrial Systems of Gabes in 2015. He is currently an Assistant at the Higher Institute of Technological Studies at Djerba. He is member at research Laboratory on Modeling, Analysis and Control of Systems (MACS). His research interests include fault tolerant control, fault diagnosis and nonlinear systems.

Mohamed Naceur Abdelkrim was born in Tunisia in 1958, he graduated from High School of Technical Education of Tunis, Tunisia, in 1980. He received the Master degree from the same school in 1981 and the $\mathrm{PhD}$ degree and "thèse d'état" from the National School of Engineers of Tunis, Tunisia respectively, in 1984 and 2003. He is currently a professor of automatic control at the National School of Engineers of Gabes. $\mathrm{He}$ is also the head of the research Laboratory on Modeling, Analysis and Control of Systems (MACS). His research interests include robust control, fault tolerant control and singularly perturbed systems. 